\title{
Random Lasing Action from PMMA Waveguide Doped with CdSe/ZnS CQDs Plasmonically Enhanced by Ag Nanoislands

\author{
Qing Zhao ${ }^{1}$, Lihua Ye ${ }^{1,2, *}$, Zhixiang Cheng ${ }^{1}$, Shaoqiang Hong ${ }^{1}$, Richard Penty ${ }^{2}$, lan
} \\ White ${ }^{2}$
}

1. Advanced Photonics Center, School of Electronic Science and Engineering, Southeast University, Nanjing 210018, P. R. China

2. Electrical Engineering Division, Department of Engineering, University of Cambridge, CAPE Building 9 JJ Thomson Avenue CB3

OFA, Cambridge UK

*Corresponding author: ylh@seu.edu.cn

Abstract: In this letter, the random laser is fabricated by an active waveguide structure constructed by covering the layer of poly methyl methacrylate (PMMA) doped CdSe/ZnS colloidal quantum dots (CQDs) onto the Ag nanoisland structures. There is a low threshold due to the excellent overlap of the plasmonic resonance of the Ag nanoislands with the photoluminescence spectrum of the CQDs and the strong confinement mechanism provided by the active waveguide layer. The performances of random laser can be tuned through the flexible fabrication of Ag nanoisland structures. The spectral evolution shows a stability of random lasing under uninterrupted laser irradiation owing to the super chemical and photo stability of CdSe/ZnS CQDs and the stable external environment for CdSe/ZnS CQDs provided by the PMMA matrix.

Keywords: random laser; colloidal quantum dots; PMMA waveguide; plasmonic resonance

\section{Introduction}

In the past decades, random lasers in disorder gain materials have attracted great interest within the scientific community [1-3]. Various disordered media have been employed to realize the random lasing emission, ranging from semiconductor powders [4] to $\pi$-conjugated polymers [5], dye-doped liquid crystals [6] and biological tissues [7]. Nowadays, colloidal quantum dots (CQDs) as a latest emerging optical material also are used to realize the random laser [8-10]. Due to the quantum confinement effect, CQDs exhibit various advantageous properties as optical gain media, including emission wavelength tunability over a wide spectral range through simply tailoring the size of CQDs, potentially low lasing threshold and temperature-insensitive lasing performance $[11,12]$.

In particular, the random lasing behaviors based on the localized surface plasmon (LSP) resonance of metallic nanoparticles attracted broad interest $[13,14]$. The LSP is a non-radiative local mode in the surface of metallic nanoparticles. It's emerged under the collective effect of SP and external electromagnetic wave. There is a high local field around the metallic nanoparticles when the frequency of the electromagnetic wave is close to the natural frequency of the free electron of the metallic nanoparticles [15]. The efficiency of many optical processes can be significantly improved by using the electric field enhancement effect of LSP in a very small area [16].

In this paper, random laser is achieved based on CdSe/ZnS CQDs in the red spectral range via a feedback scheme consisting of plasmonic scattering and waveguide confinement mechanisms providing effective optic gain channels. The waveguide is actually the layer of the gain medium, which is produced by spin-coating the polymer of poly methyl methacrylate (PMMA) doped with CdSe/ZnS CQDs onto the Ag nanoisland structures.

\section{Experiment}

The colloidal solution of $\mathrm{Ag}$ nanoparticles dispersed in toluene with a concentration of $5.62 \times 10^{-10} \mathrm{~mol} / \mathrm{L}$ was synthesized [17] and drop casted onto the clean glass substrate before solvent evaporation at room temperature for $30 \mathrm{~min}$. Thus, the Ag nanoisland structures with an area of $1.5 \times 1.5 \mathrm{~cm}^{2}$ were produced. As the image of Scanning Electron Microscope
(SEM) shows in Figure 1(a), the Ag nanoislands, which have considerably homogeneous shape and average size of about 30 $\mathrm{nm}$, were randomly distributed on the glass substrate. Then, the blend solution of CdSe/ZnS CQDs (purchased from Suzhou Xingshuo Nanotech Co., Ltd. with diameter of about $8 \mathrm{~nm}$ ) and PMMA was prepared by adding $0.34 \mathrm{~g}$ PMMA into $4 \mathrm{~mL}$ solution of CdSe/ZnS CQDs dissolved in toluene with a concentration of 5 $\mathrm{mg} / \mathrm{mL}$ and magnetic stirring $12 \mathrm{~h}$. Later, the blend solution was spin-coated onto the glass substrate at a speed of $1000 \mathrm{rpm}$. Thus, a PMMA waveguide doped with CdSe/ZnS CQDs was produced on top of the Ag nanoisland structures. The random laser device was pumped by a frequency-tripled Q-switched Nd:YAG laser (PowerLite Precision II 8010) at a wavelength of $355 \mathrm{~nm}$, a repetition rate of $10 \mathrm{~Hz}$, a pulse duration of $8 \mathrm{~ns}$. A cylindrical lens was aligned to sharpen a laser pump stripe whose length and width were 5 and $0.3 \mathrm{~mm}$, respectively. The emitting signal was along the pump stripe, and was collected by an Optical Multichannel Analyzer (OMA) with a spectral resolution of $0.1 \mathrm{~nm}$

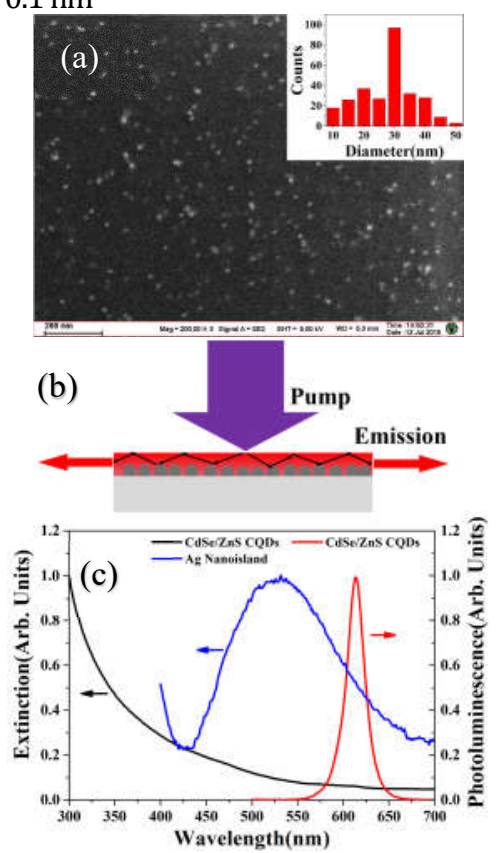

Figure 1. (a) The SEM images of the Ag nanoisland structures 
prepared on a glass substrate. The inset is the particle size distribution histogram. (b) Basic principles of the random laser device based on the waveguided plasmonic Ag nanoisland. (c) The extinction (black curve) and the photoluminescence (red curve) spectra of CdSe/ZnS CQDs dissolved in toluene and the extinction spectra (blue curve) of Ag nanoislands on the glass substrate.

The figure 1(b) illustrates the stimulation scheme with the pumping, the scattering, and the emitting, which forms the waveguided plasmonic feedback mechanism and supports stimulated emission and amplification of the CQDs radiation. The great amount of the scattered light may be reflected back totally at the PMMA-air interface to propagate within the active waveguide layer and scattered further by the Ag nanoislands, which experiences strong amplification through stimulated emission [18]. The figure 1(c) shows the extinction and the photoluminescence (PL) spectra of CdSe/ZnS CQDs dissolved in toluene and the extinction spectra of Ag nanoislands on the glass substrate. It is find that the extinction peak of $\mathrm{Ag}$ nanoislands is $532 \mathrm{~nm}$ and the PL peak of CdSe/ZnS CQDs is $613 \mathrm{~nm}$.

\section{Results and discussion}

In the first place, the sample was fabricated and pumped, where the Ag nanoisland structures were not introduced. The emission from the PMMA waveguide film doped with $\mathrm{CdSe} / \mathrm{ZnS}$ CQDs exhibits a clear transition from PL to amplified spontaneous emission (ASE) with a narrow full width at half maximum (FWHM) of $10 \mathrm{~nm}$ with the increasing pump energy, as shown in Figure 2. This phenomenon suggests that the population inversion and incoherent optical amplification are achieved $[10,19,20]$. The ASE peak is red-shifted with respect to the center of spontaneous emission by $58.17 \mathrm{meV}$. This red shift indicates a positive bi-exciton binding energy due to attractive exciton-exciton interactions, which is typically found in core/shell CQDs with a type-I band alignment [21]. The integrated intensity of ASE emission increases nonlinearly with the increasing pump energy, as shown in the inset of Figure 2, where an obvious threshold action $\left(\sim 2.0 \mathrm{~mJ} / \mathrm{cm}^{2}\right)$ is observed.

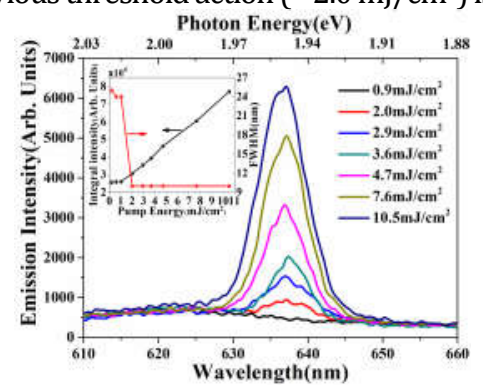

Figure 2. Spectral evolution of the ASE emissions as a function of the pump energy. The Ag nanoislands were not introduced.

When the Ag nanoisland structures were introduced in this system, the random lasing can be observed as the pump energy exceeds the threshold as shown in figure 3(a). It can be seen that the narrow discrete spikes emerge and superimpose on the corresponding ASE peaks, an evidenced signature of a random laser associated with the coherent optical feedback $[9,10,22]$. The FWHM of individual oscillation modes is about $0.2 \mathrm{~nm}$ which is much narrower than that of the ASE. The observation of oscillation modes indicates that the $\mathrm{Ag}$ nanoislands can be light scattering centers causing multiple scattering and enhance light localization through LSP resonance which can spatially confine the light near the surface to enable high gain for lasing [23]. (a)
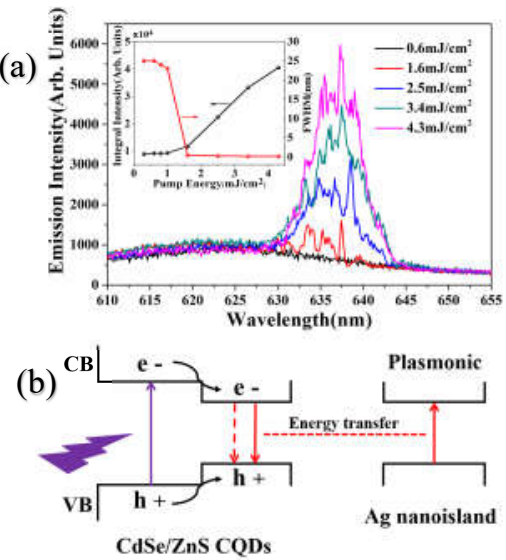

Figure 3. (a) Spectral evolution of the random laser as a function of the pump energy. The Ag nanoislands were introduced. (b) Schematics of absorbing and emitting transitions in CdSe/ZnS CQDs and plasmonic resonance energy transfer between Ag nanoisland and CdSe/ZnS CQDs.

The schematics of absorbing and emitting transitions in $\mathrm{CdSe} / \mathrm{ZnS}$ CQDs and plasmonic resonance energy transfer between Ag nanoisland and CdSe/ZnS CQDs is shown in Figure 3(b). Because the spectrum of particle plasmonic resonance overlaps the emission spectrum of the CdSe/ZnS CQDs (figure $1(c)$ ), there is a strongly coupling effect between LSP resonances of the Ag nanoislands and PL emissions of the CdSe/ZnS CQDs. This coupling effect is beneficial to the realization of high gain or population inversion. The CdSe/ZnS CQDs is pumped by an external pump light, exciting the CQDs from valence band (VB) to conduction band (CB) [24]. The electron-hole pair is excited and relaxes to an excitonic state due to carrier multiplication. In the free CQDs, the excitons would recombine to form photons. However, when the CQDs is sitting on the surface of the $\mathrm{Ag}$ nanoislands, the excitonic energy is transferred, without any significant emission of radiation, to the LSP mode of $\mathrm{Ag}$ nanoislands, a process that has a much larger probability by orders of magnitude. The LSP create the high local fields that further excite the CdSe/ZnS CQDs and stimulate more emission to this mode, which is the feedback mechanism. This plasmonic scattering not only achieved high gain due to the high local fields around the Ag nanoislands, but also provided multiple scattering and coherent feedback for random lasers emission.

The corresponding integrated intensities from 630 to 645 $\mathrm{nm}$ and FWHM of emission spectrum with respect to pumping energy are presented in the inset of Figure 3(a). The abrupt increase of the integrated intensity and the rushed decrease of FWHM indicates the achievement of random lasing with a threshold of $\sim 1.6 \mathrm{~mJ} / \mathrm{cm}^{2}$. There is a lower threshold comparing to reported random lasing based on CQDs $[8,10,21]$. The reason is that the PMMA active waveguide layer provides high quality confinement of the radiation for efficient amplification (Figure 1(b)). The random lasing results from multiple scattering for CdSe/ZnS CQDs radiation taking place sequentially by the disordered plasmonic Ag nanoislands at their interfaces with the PMMA doped with CdSe/ZnS CQDs, which is enhanced by the PMMA layer through the confinement of the scattered light into the waveguide [18]. Under the combined action of the strong scattering by the Ag nanoislands and the total reflection at the PMMA-air interface, the closed loops are formed for coherent optical feedback. Thus, each closed loop corresponds to a gain process through a scattering-total reflection-scattering process and the lasing is emitted when the gain overcome the loss in this 
process.

For the random laser, its action depends strongly on the minimum mean free path length, which is defined as $l_{\min }=2 n^{2} d /\left(n^{2}-1\right)^{1 / 2}$, where $\mathrm{n}$ and $\mathrm{d}$ are the refractive index and the thickness of the gain medium, respectively [18]. In our experiments, when $\mathrm{d}$ is $1.8 \mu \mathrm{m}$, the $l_{\min } \approx 7.2 \mu \mathrm{m}$ is obtained. This path length is multiplied by the total reflection process and extended within the waveguide. Thus, the separation between the Ag nanoislands, required by this minimum mean free path length, must be smaller than $S=l_{\min } \sin \theta_{c}$, where $\theta_{c}=\sin ^{-1}(1 / n)$ is the critical angle for total reflection at the PMMA-air interface. So, the $S \approx 4.8 \mu \mathrm{m}$ is obtained. As the SEM image shown in Figure 1(a), the separation between the Ag nanoislands is actually smaller than the value of $\mathrm{S}$. Therefore, the $\mathrm{Ag}$ nanoisland structures in this system facilitate the achievement of multiple scattering and coherent feedback for random lasers.

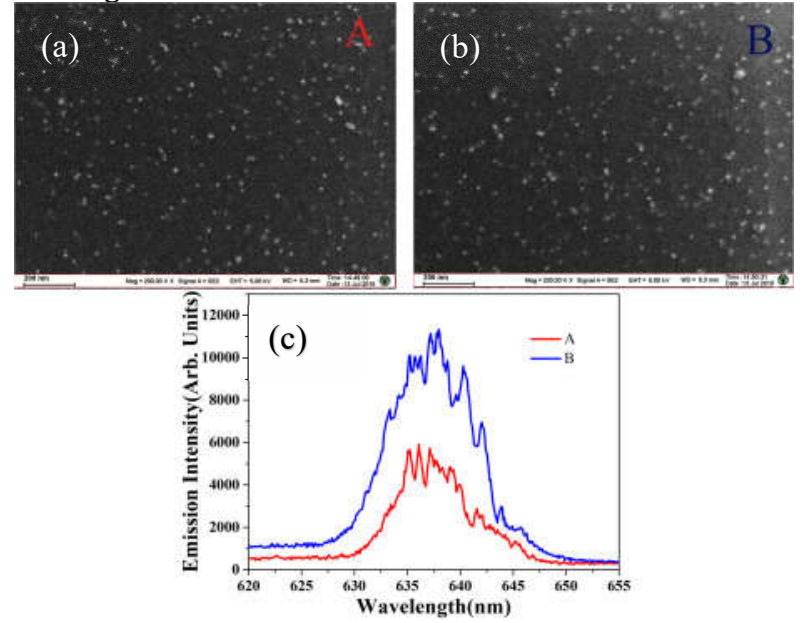

Figure 4. The SEM images of the Ag nanoisland structures with different densities (a: sample A with $\sigma_{A}=1.5 \times$ $10^{6} \mathrm{~cm}^{-2}$ and b: sample B with $\sigma_{B}=2.25 \times 10^{6} \mathrm{~cm}^{-2}$ ) and (c) the emission spectrums of random lasing for sample $A$ and sample B.

In order to further determine the role of $\mathrm{Ag}$ nanoisland structures in the random laser, the different concentrations of Ag nanoparticles were used to form $\mathrm{Ag}$ nanoislands with different densities, $\sigma_{A}=1.5 \times 10^{6} \mathrm{~cm}^{-2}$ and $\sigma_{B}=2.25 \times 10^{6} \mathrm{~cm}^{-2}$. The SEM images are shown in Figure 4(a) and (b). The emission spectrums of random lasing were recorded when the pump energy was $4.4 \mathrm{~mJ} / \mathrm{cm}^{2}$ (Figure 4(c)). It can be found that the emission intensity and oscillation modes increase when the density of Ag nanoislands increases. The increase of plasmon resonance, when the density of Ag nanoislands increases, lead to the increase of scattering strength. Further, that causes the decreasing of mean free path and the forming of more closed loops. Therefore, more oscillation modes appear in the emission spectrum. Moreover, the interaction between CdSe/ZnS CQDs and $\mathrm{Ag}$ nanoislands also is increased with the increase of density of Ag nanoislands, which bring about the enhanment of radiation from the CdSe/ZnS CQDs and the increase of emission intensity. Thus, the Ag nanoisland structures fabricated by drop casted the solution of Ag nanoparticles onto the glass substrate play the role of enhanced scattering due to the LSP resonance effect. And the random laser can be easily tuned by changing the density of Ag nanoislands, which can be achieved by modifying the concentration of the solution of Ag nanoparticles during the fabrication process.

According to the random lasing theory, there is a critical area for the formation of ring cavities [1]. When the pumping area is below this value, there is not enough space for the formation of closed loops. More and more closed loops can find sufficient space to develop in the disordered system, accompanied by more and more spikes appearing in the emission spectra as the excitation area keeps increasing $[8,25,26]$. As shown in Figure 5(a), the number of lasing spikes and the corresponding intensities increase significantly when the pump stripe length increases from 3.0 to $9.0 \mathrm{~mm}$. It can be found that no lasing action occur while the pump stripe length is less than $5.0 \mathrm{~mm}$. These observations confirm that the dominant mechanism is based on multiple light scattering associated with the LSP resonances of the Ag nanoislands in this system, because there exists a critical gain volume, above which the spontaneous emission converts into random lasing action with distinct spikes growing rapidly [10]. When the excitation region is larger than the critical volume, since there are more closed loop paths for light in a larger excitation volume, laser action could occur in more cavities formed by recurrent scattering [1]. On the other hand, When the excitation region is smaller than the critical volume, since the closed loop paths is too short, the gain along the loops is not high enough to achieve lasing.
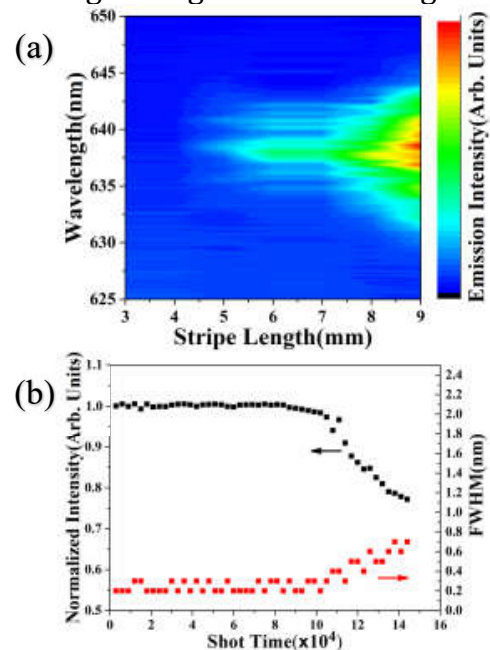

Figure 5. (a) Spectral evolution with increasing pump stripe length from 3.0 to $9.0 \mathrm{~mm}$, and (b) plot of integrated intensity and FWHM of random lasing as a function of laser shoots. The pump energy was $2.0 \mathrm{~mJ} / \mathrm{cm}^{2}$.

Moreover, the random lasing from this system shows an excellent stability upon uninterrupted laser irradiation. Figure 5(b) displays the integrated intensity and FWHM as a function of laser shots under pump intensity of $2.0 \mathrm{~mJ} / \mathrm{cm}^{2}$. The integrated intensity and the FWHM could hold good stability in the $10^{5}$ laser shots $(\sim 3 \mathrm{~h})$, thanks to the stable external environment for CdSe/ZnS CQDs provided by the PMMA matrix and the super chemical and photo stability of CdSe/ZnS CQDs $[27,28]$.

\section{Conclusions}

In conclusion, the random lasing action is observed from the scheme comprised by a bottom layer of randomly distributed Ag nanoislands on the glass substrates and a top layer of active waveguide made of PMMA matrix doped with $\mathrm{CdSe} / \mathrm{ZnS}$ CQDs. The pump threshold is only $1.6 \mathrm{~mJ} / \mathrm{cm}^{2}$ because the free path length is extended by the strong plasmoinc scattering of Ag nanoislands and the strong confinement of the active waveguide layer. In addition, the emission intensity and oscillation modes of the random laser can be tuned by changing the density of Ag nanoislands achieved by modifying the concentration of the solution of Ag nanoparticles during the 
fabrication process. Moreover, there exists a critical gain volume, above which the spontaneous emission converts into random lasing action with distinct spikes growing rapidly as the increasing of pump stripe length. Furthermore, the random lasing action shows an excellent stability due to the super chemical and photo stability of CdSe/ZnS CQDs and the stable external environment for CdSe/ZnS CQDs provided by the PMMA matrix. This kind of random laser has prospects in a wide range of applications, such as biomedical diagnosis [29], display imaging [30] and environment lighting [31].

\section{Acknowledgments}

This work was supported by Jiangsu Overseas Visiting Scholar Program for University Prominent Young \& Middle-aged Teachers and Presidents under No.20180013 and the National Natural Science Foundation of China under Grant No.11174160, Grant No.11474052 and Grant No.11274062.

\section{Declarations of interest: none}

\section{References}

[1] H. Cao, Y. Zhao, S. Ho, E. Seelig, Q. Wang, R. Chang, Random Laser Action in Semiconductor Powder, Physical Review Letters 82 (1999) 2278.

[2] J. Fallert, R. Dietz, J. Sartor, D. Schneider, C. Klingshirn, H. Kalt, Co-existence of strongly and weakly localized random laser modes, Nature Photonics 3 (2009) 279.

[3] N. Bachelard, S. Gigan, X. Noblin, P. Sebbah, Adaptive pumping for spectral control of random Lasers, Nature Physics 10 (2014) 426.

[4] E. Chelnokov, N. Bityurin, I. Ozerov, W. Marine, Random lasing from dye-gold nanoparticles in polymer films: Enhanced gain at the surface-plasmon-resonance wavelength, Applied Physics Letters 89 (2006) 171119.

[5] A. Tulek, R. Polson, Z. Vardeny, Naturally occurring resonators in random lasing of $\pi$-conjugated polymer films, Nature Physics 6 (2010) 303.

[6] S. Perumbilavil, A. Piccardi, R. Barboza, O. Buchnev, M. Kauranen, G. Strangi, G. Assanto, Beaming random lasers with soliton control, Nature Communications 9 (2018) 3863.

[7] R. Polson, Z. Vardeny, Random lasing in human tissues, Applied Physics Letters 85 (2004) 1289.

[8] Y. Chen, J. Herrnsdorf, B. Guilhabert, Y. Zhang, I. Watson, E. Gu, N. Laurand, M. Dawson, Colloidal quantum dot random laser Optics Express 19 (2011) 2996.

[9] Y. Wang, V. Ta, Y. Gao, T. He, R. Chen, E. Mutlugun, H. Demir, $\mathrm{H}$. Sun, Stimulated Emission and Lasing from CdSe/CdS/ZnS CoreMulti-Shell Quantum Dots by Simultaneous Three-Photon Absorption, Advanced Materials 26 (2014) 2954.

[10] Y. Yao, Z. Yang, J. Hwang, H. Su, J. Haung, T. Lin, J. Shen, M. Lee, M. Tsai, Y. Lee, Coherent and Polarized Random Laser Emissions from Colloidal CdSe/ZnS Quantum Dots Plasmonically Coupled to Ellipsoidal Ag Nanoparticles, Advanced Optical Materials 5 (2016) 1600746.

[11] C. Dang, J. Lee, C. Breen, J. Steckel, S. Coe-Sullivan, A. Nurmikko, Red, green and blue lasing enabled by single-exciton gain in colloidal quantum dot films, Nature Nanotechnology 7 (2012) 335.

[12] P. Kambhampati, Multiexcitons in Semiconductor Nanocrystals: A Platform for Optoelectronics at High Carrier Concentration, The Journal of Physical Chemistry Letters 3 (2012) 1182.

[13] O. Popov, A. Zilbershtein, D. Davidov, Random lasing from dye-gold nanoparticles in polymer films: Enhanced gain at the surface-plasmon-resonance wavelength, Applied Physics Letters 89 (2006) 191116.

[14] X. Meng, K. Fujita, Y. Zong, S. Murai, K. Tanaka, Random lasers with coherent feedback from highly transparent polymer films embedded with silver nanoparticles, Applied Physics Letters 92 (2008) 201112.

[15] K. Kelly, E. Coronado, L. Zhao, G. Schatz, The Optical Properties of Metal Nanoparticles: The Influence of Size, Shape, and Dielectric Environment, J. Phys. Chem. B 107 (2003) 668.

[16] R. Hillenbrand, T. Taubner F. Keilmann, Phonon-enhanced light-matter interaction at the nanometre scale, Nature 418 (2002) 159.

[17] L. Ye, B. Liu, F. Li, Y. Feng, Y. Cui, Y. Lu, The influence of Ag nanoparticles on random laser from dye-doped nematic liquid crystals Laser Physics Letters 13 (2016) 105001.

[18] T. Zhai, X. Zhang, Z. Pang, X. Su, H. Liu, S. Feng, L. Wang, Random Laser Based on Waveguided Plasmonic Gain Channels Nano Letters 11 (2011) 4295.

[19] C. Li, Z. Zang, C. Han, Z. Hu, X. Tang, J. Du, Y. Leng, K. Sun, Highly compact $\mathrm{CsPbBr}_{3}$ perovskite thin films decorated by $\mathrm{ZnO}$ nanoparticles for enhanced random lasing, Nano Energy 40 (2017) 195.

[20] S. Yakunin, L. Protesescu, F. Krieg, M. I. Bodnarchuk, G. Nedelcu, M. Humer, G. Luca, M. Fiebig, W. Heiss, M. V. Kovalenko, Low-threshold amplified spontaneous emission and lasing from colloidal nanocrystals of caesium lead halide perovskites, Nature Communications 6 (2015) 8056.

[21] C. Gollner, J. Ziegler, L. Protesescu, D. Dirin, R. Lechner, G. Fritz-Popovski, M. Sytnyk, S. Yakunin, S. Rotter, A. Yousefi Amin, C. Vidal, C. Hrelescu, T. Klar, M. Kovalenko, W. Heiss, Random Lasing with Systematic Threshold Behavior in Films of CdSe/CdS Core/Thick-Shell Colloidal Quantum Dots, ACS Nano 9 (2015) 9792.

[22] X. Li, Y. Wang, H. Sun, and H. Zeng, Amino-Mediated Anchoring Perovskite Quantum Dots for Stable and Low-Threshold Random Lasing, Advanced Materials 29 (2017) 1701185.

[23] F. Luan, B. Gu, A. Gomes, K. Yong, S. Wen, P. Prasad, Lasing in nanocomposite random media, Nano Today 10 (2015) 168.

[24] M. Stockman, Spasers explained, Nature Photonics 2 (2008) 327.

[25] H. Hsu, C. Wu, W. Hsieh, Stimulated emission and lasing of random-growth oriented ZnO nanowires, Journal of Applied Physics 97 (2005) 064315.

[26] X. Meng, K. Fujita, S. Murai, K. Tanaka, Coherent random lasers in weakly scattering polymer films containing silver nanoparticles, Physical Review A 79 (2009) 053817.

[27] Q. Sun, Y. Wang, L. Li, D. Wang, T. Zhu, J. Xu, C. Yang, Y. Li, Bright, multicoloured light-emitting diodes based on quantum dots, Nature Photonics 1 (2007) 717.

[28] Y. Yang, Y. Zheng, W. Cao, A. Titov, J. Hyvonen, J. Manders, J. Xue, P. Holloway, L. Qian, Highefficiency light-emitting devices based on quantum dots with tailored nanostructures, Nature Photonics 9 (2015) 259.

[29] Y. Wang, Z. Duan, Z. Qiu, P. Zhang, J. Wu, D. Zhang, T. Xiang, Random lasing in human tissues embedded with organic dyes for cancer diagnosis, Scientific Reports 7 (2017) 8385.

[30] S. Gottardo, S. Cavalieri, O. Yaroshchuk, D. Wiersma, Quasi-Two-Dimensional Diffusive Random Laser Action, Physical Review Letters 93 (2004) 263901.

[31] D. Wiersma, The physics and applications of random lasers, Nature Physics 4 (2008) 359. 
